\title{
Ein gemeyn leycht buechlein
}

\author{
Zur Didaktik in Adam Ries' zweitem Rechenbuch \\ im Vergleich zu Widmanns „Behende vnd hubsche \\ Rechenung"
}

Peter Gabriel

Ein gemeyn leycht buechlein. On the Didactics of Adam Ries' Second Arithmetic Textbook in Comparison with Widmann's "Behende vnd hubsche Rechenung"

Adam Ries wrote the most popular German textbook on arithmetics in Early Modern History, Rechenung auf der linihen vnd federn (Calculations with the Computing Table and with Numerals). This contribution takes a systematic look at the didactic benefits of Ries' book by comparing it with the contemporary textbook by Johannes Widmann. The analysis covers three levels: the mathematical content of both textbooks, the design of their main text unitsexplanations and exercises - as well as the specific utilization of grammar, vocabulary, and notational schemes by Ries and Widmann. In contrast to Widmann, Ries tailored his book to the needs of his audience. He selected and structured the mathematical content according to the practical needs of merchants and craftsmen without denying traditions like the abacus-style calculating. His explanations and exercises followed the usual rules of arithmetic textbooks but showed great flexibility in adapting to the learning process of the reader. This was supported by a stereotypical linguistic style that helped the reader to get through the text quickly.

Keywords: Adam Ries, Johannes Widmann, arithmetic, textbook, didactics

Schlüsse/wörter: Adam Ries, Johannes Widmann, Arithmetik, Rechenbuch, Didaktik

Die Kunst des Rechnens war im deutschen Spätmittelalter eine noch wenig verbreitete Fähigkeit. Das änderte sich mit dem wachsenden Bedarf von Kaufleuten und Handwerkern rechnen zu können, und dem beginnenden Aufbau eines allgemeinen Schulsystems. Einen großen Anteil an der Verbreitung der praktischen Arithmetik im deutschsprachigen Raum hatten die Rechenschulen und Rechenbücher des 15. und 16. Jahrhunderts. Der herausragende Autor war dabei der erzgebirgische Rechenmeister Adam Ries (1492-1559), der zunächst in Erfurt und danach in der Bergbaustadt Annaberg Rechenschulen unterhielt und gleichzeitig wichtige Ämter in der kursächsischen Bergbauverwaltung einnahm (Wußing 2009, Deubner 1992). Sein zweites Rechenbuch Rechenung auf der linihen vnd federn (1522) ließ mit 113 belegten Auflagen die zahlreichen anderen Rechenbücher der Zeit in der 
Verbreitung weit hinter sich (Gebhardt 2003: 37-40). Ries wurde selbst für die Zeitgenossen so sehr zum Inbegriff des Rechenmeisters, dass ab den 1550er Jahren sein Portrait - einzigartig für diese Art von Texten - auf vielen Neuauflagen seiner Rechenbücher abgedruckt wurde (Wolff-Thomsen 2001: 189, vgl. Abb. 1).

Womit hob sich Ries' zweites Rechenbuch von anderen Examplaren dieser Gattung seiner Zeit ab? Die Literatur zu Ries verweist in der Regel auf einzelne pädagogische Aspekte, etwa den besonders gut geeigneten fachlichen Aufbau des Buchs und die große Menge an Übungsaufgaben (Unger 1888: 51-52, Wußing 2009: 61-63, Deschauer 1992: 9-10, 2003: 27-28). Eine sprachwissenschaftliche Betrachtung hat Gärtner (2000) in ihrer germanistischen

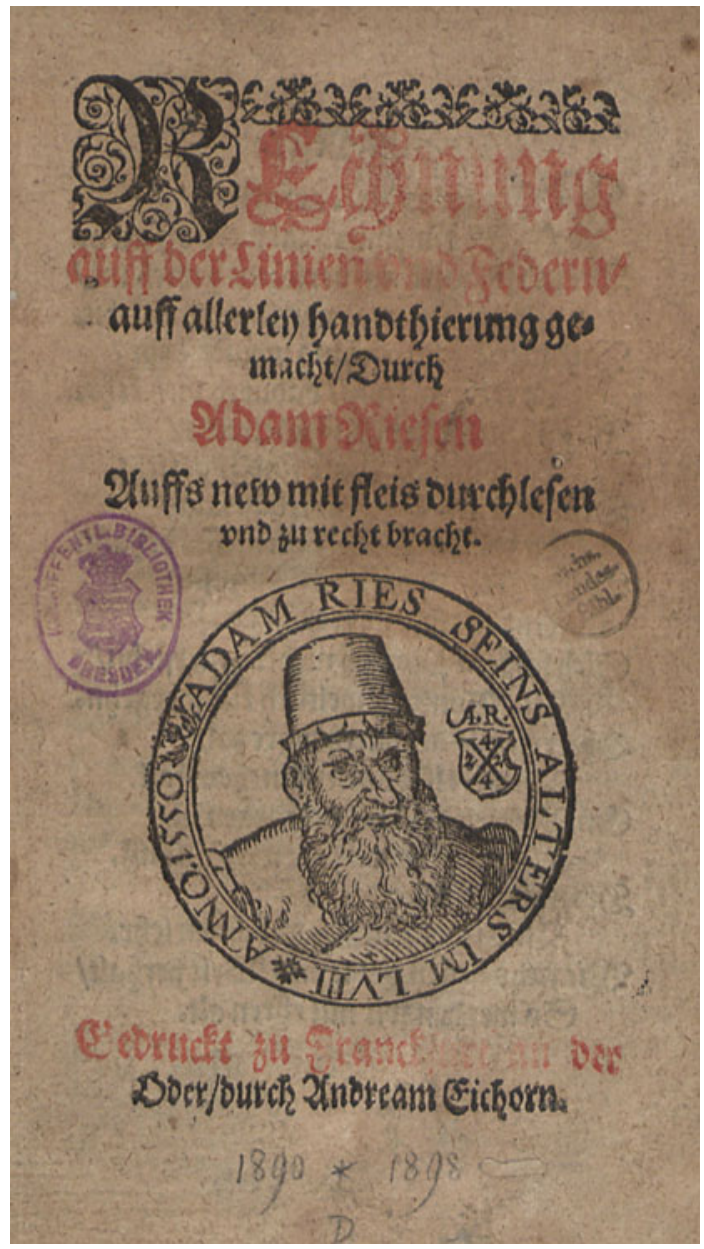

Abb. 1 Porträt von Adam Ries auf dem Titelbild seines zweiten Rechenbuchs Rechenung auf der linihen vnd federn von 1522 (SLUB Dresden, http://digital.slub-dresden.de/ppn266 980112, [9.9.2010]) 
Untersuchung des Rechenbuchs Behende vnd hubsche Rechenung von Johannes Widmann vorgenommen, wo sie Aufbau und Sprachstil der Rechenbücher von Widmann und Ries vergleicht. Eine tiefergehende systematische Analyse von Ries' Didaktik hat bisher in den meist mathematik- und wissenschaftshistorisch ausgerichteten Arbeiten jedoch nicht stattgefunden. In diesem Beitrag soll die didaktische Methodik von Adam Ries' Rechenung auf der linihen vnd federn näher untersucht werden. Um die pädagogischen Besonderheiten dieses Werks erkennen zu können, bedarf es eines Vergleichsmaßstabs. Dazu soll das 1489 erschienene Rechenbuch Behende vnd hubsche Rechenung auf allen kaufmannschaft des böhmischen Mathematikers Johannes Widmann (1460?-1500?) dienen. Von Widmanns Leben ist wenig bekannt, außer dass er an der Universität Leipzig studierte und dort die erste bekannte Algebra-Vorlesung in Deutschland hielt (Kaunzner 1996). Mit immerhin fünf Auflagen war Widmanns Werk eines der populären frühen Rechenbücher um 1500 und hatte, wie Ries' Buch, den Anspruch, Kaufleute und Handwerker das Rechnen zu lehren. Ries kannte Widmanns Rechenbuch, schätzte dessen pädagogische Eignung aber nur als gering ein. Im Vorwort seines zu Lebzeiten unveröffentlichten Algebra-Manuskripts Coß gesteht Ries Widmanns Buch nur ,an wenigk ortenn eine rechte vnderweisung“ zu (Gebhardt 1994: 18). Die Rechenung auf der linihen vnd federn sollte anders als die Behende vnd hubsche Rechenung tatsächlich ein „gemeyn leycht buechlein [...] fur iunge anhebende schuler" sein (Ries 1522: fol. $\mathrm{A}^{2 \mathrm{r}}$ ).

Bezugspunkte des didaktischen Vergleichs der Rechenbücher von Ries und Widmann sind die jeweils ersten Auflagen, ohne dass dies eine Einschränkung darstellt. Auch wenn in den späteren Auflagen Fehler korrigiert sowie orthographische und typographische Änderungen vorgenommen wurden, blieben beide Bücher ansonsten weitgehend unverändert (Deschauer 1991 [1522]: 9-11, Gärtner 2000: 54-57). Widmanns Buch bietet jedoch deutlich mehr Lehrstoff als das von Ries. Der Vergleich beschränkt sich daher weitgehend auf die Abschnitte der Behende vnd hubsche Rechenung, zu denen es ein inhaltliches Gegenstück in der Rechenung auf der linihen vnd federn gibt.

Meine Untersuchung beginnt mit einer Betrachtung des Umfelds für die weiteren Untersuchungen: Was umfasste die Mathematik der Renaissance? Was davon benötigten die deutschen Kaufleute und Handwerker der frühen Neuzeit? Wie sah ihr Rechenunterricht aus, und welche Rolle spielten dabei das Rechenbuch, eine der frühen und von Anfang an populären Textsorten der deutschsprachigen Fachprosa? Der didaktische Vergleich der Rechenbücher von Ries und Widmann erfolgt dann auf drei Ebenen: Zunächst werden die mathematischen Inhalte der beiden Rechenbücher und ihre jeweilige Anordnung betrachtet. Danach folgt eine Analyse der zwei wichtigsten Typen von Lehrtexten in den beiden Büchern: die Erläuterungen von Rechenarten und -regeln und die Übungsaufgaben. Den Abschluss bildet schließlich eine Betrachtung der sprachlichen Gestaltung und des Einsatzes von Notationsschemata in den beiden Rechenbüchern. 


\section{Arithmetik, Rechenunterricht und Rechenbuch in der frühen Neuzeit}

Das mathematische Wissen im Europa des 14. und 15. Jahrhunderts speiste sich aus zwei Quellen. Für die ältere, griechisch-römische Tradition stand vor allem die Arithmetik des römischen Konsuls und Gelehrten Boethius (ca. 475-524), die etwa in der weit verbreiteten Arithmetica des Mathematikers Jordanus Nemorarius (frühes 13. Jahrhundert) fortgeführt wurde. Bedeutender war jedoch, dass arabische Autoren ebenfalls die griechischen Grundlagen übernommen und eigenständig erweitert hatten. Von ihnen stammten das indische Dezimalystem und dessen Arithmetik sowie große Teile der Algebra des europäischen Mittelalters und der frühen Neuzeit. Eingeleitet wurde dieser „Wendepunkt“ der europäischen Mathematik (Busard 1997: 211) durch lateinische Übersetzungen der Algebra-Schrift des persischen Gelehrten al-Hwarizmi (ca. 780-850) im 12. Jahrhundert. Insbesondere der italienische Mathematiker Leonardo de Pisa (ca. 1180-1241) popularisierte dann das indisch-arabische Zahlensystem und die algebraische Kenntnisse der Araber im christlichen Europa (ebd.: 211-128). Leonardos Buch Liber Abbaci (1202, Überarbeitung 1228) wurde zum Vorbild und zur Quelle italienischer und deutscher Rechenbücher (Folkerts/Reich: 2001: 189-194; Übersichten zur Entwicklung der Mathematik im späten Mittelalter und in der frühen Neuzeit geben Alten u. a. 2003, Gericke 1980, Wußing 2008).

Die Arithmetik der frühen Neuzeit bezog sich in der Regel auf die ganzen und die gebrochenen Zahlen. Rechenarten waren Addieren, Duplieren (= Verdoppeln), Subtrahieren, Multiplizieren, Medieren (= zur Hälfte nehmen), Dividieren und Potenzieren (Unger 1888: 72). Für das Ziehen von quadratischen und kubischen Wurzeln waren schon Näherungsverfahren bekannt (ebd.: 99). Negative Zahlen wurden von manchen Autoren bereits als Schulden interpretiert und waren damit auch als Zahlen zugelassen (Wußing 2008: 317). Die tatsächliche Existenz der irrationalen Zahlen, die etwa durch das Wurzelziehen entstehen, war dagegen noch lange umstritten (Gericke 1980: 245246).

Die gelehrten Mathematiker verwendeten in der Regel längst die arabischen Ziffern. Die übrige Bevölkerung notierte die Zahlen dagegen häufig noch in den römischen Ziffern, die im deutschsprachigen Raum geradezu als ,deutsche Ziffern' galten (Unger 1888: 13-15). Oft wurde noch mit dem Rechentisch oder dem Rechentuch ,auf den Linien' gerechnet, ein System das sich auch mit den römischen Ziffern vertrug und bei nicht im Kopf gerechnet werden musste (ebd.: 66-68). ${ }^{1}$ Rationale Zahlen wurden als Quotient von Zähler und Nenner geschrieben und bereits mit einem Bruchstrich notiert. Die Darstellung als Dezimalbruch wurde endgültig erst zum Ende des 16. Jahrhunderts zum Allgemeingut. 
Rechnungen und Rechenaufgaben wurden noch weitgehend verbal formuliert. Allerdings verbreiteten sich - wie erwähnt, meist basierend auf arabischen Werken - seit dem 12. Jahrhundert auch in Westeuropa weiterreichende algebraische Kenntnisse. Bestimmte Gleichungstypen wurden geometrisch interpretiert und gelöst. Zudem etablierten sich für Unbekannte und ihre Potenzen sowie für Operatoren und Gleichheitszeichen nach und nach zunächst feststehende Begriffe und später Symbolzeichen (Wußing 2008: 322-326). Das machte es allmählich möglich, Gleichungen auch algebraisch umzuformen und zu lösen - ein Prozess, der allerdings erst 1591 mit dem französischen Juristen und Mathematiker François Viète (1540-1603) zum Abschluss kam (ebd.: 266-273). In Deutschland wurde die frühe Form der Algebra als ,Coß’ bezeichnet (nach dem italienischen cosa als Bezeichnung für die Unbekannte in einer Gleichung, ebd.: 331-334). Weitaus gebräuchlicher als diese nur wenigen zugängliche Coß waren jedoch andere Lösungswege: Für den Dreisatz (regula de tri oder regula detri) und daraus abgeleitete Rechenregeln wie die Zinsrechung gab es feste Lösungswege (Unger 1888: 86-92). Rechenaufgaben mit Unbekannten - lineare Gleichungen im heutigen Verständnis - wurden mittels des ,doppelten falschen Ansatzes' (regula falsi) gelöst (Tropfke 1980: 180-185). ${ }^{2}$ Für bestimmte Gleichungen höherer Ordnung gab es gleichfalls feste Lösungsregeln, die nach arabischem Vorbild geometrisch hergeleitet wurden (Wußing 2008: 278).

Bis in das 14. Jahrhundert hinein wurde in Deutschland die Arithmetik, ebenso wie die Geometrie, vor allem in den kirchlichen Schulen und den Universitäten gelehrt und weiterentwickelt (Günther 1969 [1887]: 127-141, 192-200). Für die Kaufleute des deutschen Frühkapitalismus war dieses Angebot jedoch nicht länger ausreichend. Steigende Handelsumsätze, die zunehmende Bedeutung des Fernhandels sowie die Einführung von Gesellschaften und Verlagen als neue Formen wirtschaftlicher Kooperationen erforderten den Übergang von der Tausch- zur Geldwirtschaft und die Nutzung des Kredits als neues Zahlungs- und Finanzierungsinstrument. Diese Entwicklung begann zunächst in den oberdeutschen Handelsstädten und dehnte sich schnell nach Norden aus. Vorläufer und Vorbild der deutschen Kaufleute waren ihre italienischen Kollegen, bei denen Kredit- und Geldwirtschaft bereits im 13. Jahrhundert fest etabliert waren (Isenmann 2004: 503-505). Auch in einzelnen Zweigen des Handwerks, insbesondere bei Bergbau, Metallgewerbe und Tuchproduktion, wurden Kapital und Kredit zu wichtigen Produktionsmitteln und die zünftigen Handwerker zu HandwerkerUnternehmern: Bergwerke wurden von Bergbaugesellschaften betrieben, Tuchverleger beschäftigten lohnabhängige Weber, Anlagen für die Metallherstellung und -verarbeitung wurden über Kredite vorfinanziert (Isenmann 1988: 346-352). Geld- und Kreditwirtschaft setzten bei den Kaufleuten und Handwerker-Unternehmern jedoch umfangreiche Rechenkenntnisse voraus. Daneben war auch für einzelne traditionelle Gewerke die Rechenkunst 
notwendig: Münzpräger mussten die Anteile in Legierungen bestimmen, Fassmacher das Volumen von Fässern ermitteln und Bauleute geometrische Strecken und Flächen berechnen.

All dies verlangte nach spezifischen arithmetischen Kenntnissen. Zum Teil rechneten die Kaufleute und Handwerker bereits mit den arabischen Ziffern und in schriftlicher Form. Die römischen Zahlen und das Linienrechen hatten gleichwohl noch lange Bestand. Im Mittelpunkt ihrer Rechenkunst standen der Dreisatz und seine zahlreichen Varianten wie Kettenrechnung, Zinsrechnung und Gesellschaftsrechnung für die Berechnung von Anteilen und Gewinnen in Kaufmanns- und Bergwerksgesellschaften. Dazu kam häufig die aus Italien stammende, Welsche Praktik' für Aufgaben mit mehrsortigen Währungs- und Gewichtseinheiten, bei der die Beträge in rechengünstige Faktoren zerlegt wurden (Tropfke 1980: 364-365).

In den Stifts-, Kloster- und Domschulen der Kirche, aber auch in den städtischen Lateinschulen wurde jedoch noch überwiegend das mittelalterliche Trivium aus Grammatik, Logik und Rhetorik unterrichtet. Der Unterricht zielte zunächst vor allem darauf ab, die Schüler für die Beteiligung am Kirchendienst vorzubereiten. Ziel des Rechenunterrichts in den Kirch- und Lateinschulen war es lange Zeit lediglich, dem Schüler die Berechnung der beweglichen Feiertage, insbesondere des Osterfests zu ermöglichen. Der praktisch orientierte Bedarf der städtischen Kaufleute und Handwerker ging weit über dieses Angebot hinaus und wurde ab dem 15. Jahrhundert vor allem in den neu entstehenden ,deutschen', das heißt deutschsprachigen Schulen befriedigt. Die deutschen Schulen waren als private Unternehmen, zum Teil sogar zunftmäßig organisiert. Neben Lesen, Schreiben und Religion wurden hier ausgewählte Inhalte des antiken und mittelalterlichen Lehrkanons der Sieben Freien Künste (= Trivium plus Arithmetik, Geometrie, Musik und Astronomie) und besondere Berufsfertigkeiten unterrichtet. Zum Teil waren die deutschen Schulen aber auch nur ergänzende Schreib- und Rechenschulen, die bei Bedarf parallel zur Kirch- und Lateinschule besucht wurden. Den Schwerpunkt dieser Entwicklung bildeten zunächst die Zentren des Frühkapitalismus in Süddeutschland, insbesondere die fränkischen Reichsstädte und hier vor allem Nürnberg, wo ab dem 15. Jahrhundert zahlreiche Rechenschulen entstanden (Endres 1996: 375379, Hanschmidt 2005: 21-26, Endres 1982: 55-62).

Der Unterricht in den deutschen Schulen wurde von Schreib- und Rechenmeistern erteilt, deren Stand und Ansehen äußerst unterschiedlich ausfielen. Die Spannweite reichte vom fahrenden Mietschreiber über den angesehenen Rechenmeister mit einer zünftigen Ausbildung bis hin zum Magister einer Universität. Schüler waren vor allem die Kinder von Kaufleuten und Handwerkern, die ihren Vätern im Beruf folgen sollten und dafür rechnen lernen mussten (Folkerts 2003, Endres 1982). Vorbilder der deutschen Rechenmeister und ihrer Rechenschulen waren die italienischen maestri d'abbaco, die bereits seit dem 13. Jahrhundert den Kaufleuten in Italien 
volkssprachlichen Mathematikunterricht erteilten. Die Inhalte dieses Unterrichts wurden in zahlreichen Manuskripten und später auch gedruckten Büchern (libri d'abbaco) festgehalten, die, soweit heute rekonstruierbar, entweder als Unterrichtsgrundlage von den Rechenlehrern oder als Notizbücher von ihren Schülern verfasst wurden (van Egmond 1980: 27-31, Folkerts/Reich 2001: 189-194).

Vom Rechenunterricht in den deutschen Schulen und den Rechenschulen ist wenig bekannt. Sehr wahrscheinlich stand die Unterrichtsmethodik trotz des anders gearteten Inhalts noch stark in der dogmatischen Tradition der Kirchen- und Lateinschulen, in denen ein fester Kanon von Texten vom Lehrer vorgelesen und erläutert wurde, woraufhin die Schüler das Gehörte wiederholten und es kommentierten (Osterwalder 2004: 639-644). Soweit es sich, vor allem aus den Titelbildern einiger Rechenbücher, rekonstruieren lässt, trug der Rechenmeister zunächst Erläuterungen und Rechenaufgaben vor, während die Schüler mitschrieben oder im gegebenenfalls vorhandenen Rechenbuch mitlasen. Anschließend rechnete jeder Schüler für sich Aufgaben, während der Lehrer einzelne Schüler abhörte oder ihnen bei den Beispielaufgaben half (Wolff-Thomsen 2001: 185-186). Grundlage des Unterrichts in den Rechenschulen waren selbst geschriebene Rechenhefte der Lehrer oder eben eines der zahlreichen deutschsprachigen Rechenbücher, die im Buchdruckverfahren hergestellt wurden.

Mit der Einführung des Papiers in Europa im 12. Jahrhundert, insbesondere aber mit der Erfindung des Buchdrucks mittels beweglicher Lettern aus Metall um 1450 durch den Mainzer Drucker Johannes Gutenberg (um 14001468) hatte sich der Wandel vom „mittelalterlich geprägten zum neuzeitlichen Buch" (Wittmann 1999: 28) vollzogen. Auf Basis des neuen Druckverfahrens stieg die schon in der Handschriftenproduktion hohe Zahl der veröffentlichten Schriften und Bücher nochmals stark an (Neddermeyer 1998: 388). Zwischen Autor und Leser traten nun der Drucker und Verleger als Unternehmer mit dem kommerziellen Interesse, möglichst viele Werke zu publizieren (Giesecke 2006: 483-488). Schnell erkannten diese den Bedarf des städtischen Bürgertums, Fachwissen nicht länger nur in mündlicher Unterrichtung oder aus eigener Anschauung, sondern auch in schriftlicher Form zu erwerben. Damit etablierte sich im Buchdruck neben den zunächst dominierenden traditionellen theologischen, literarischen und gelehrten Textsorten die Fachprosa für den Laien, den ,gemeinen Mann'. Behandelt wurden die sieben Künste des Triviums und des Quadriums, die verbotenen Künste der Magie, der Wahrsagung und des Betrugs sowie das Recht. Vor allem aber widmete sich die Fachprosa den praktischen artes mechanicae wie Handwerk, Kriegswesen, Handel, Seefahrt, Heilkunde, Haushaltsführung und Botanik (Eis 1967: 1, Haage/Wegner 2007: 16-20).

Insbesondere die artes mechanicae sollten dem Leser nicht abstraktes Wissen vermitteln, sondern möglichst konkrete Handlungsanweisung in 
praktischen Belangen geben. Um das oft lateinunkundige städtische Bürgertum tatsächlich zu erreichen, verlegten sich von Anfang an viele Bücher der Fachprosa vom traditionellen Latein der Gelehrten auf das volkssprachliche Deutsch. Die Inhalte wurden zunächst noch von lateinischen Werken übernommen, wenn diese nicht gleich direkt in das Deutsche übersetzt wurden. Je nach Fachgebiet wurden dabei auch die lateinischen Fachbegriffe übernommen. Starke lateinische Einflüsse waren etwa im Fachvokabular der medizinischen Werke gegeben, während beispielsweise bei Bergbautexten deutsche Begriffe die Überhand hatten (Haage/Wegener 2007: 34-35). Die Syntax der deutschen Fachprosa war im Gegensatz zu den gelehrten Werken in Latein einfach und überwiegend in Satzreihungen (Parataxe) gehalten. Dies ist zumindest für naturkundlich-medizinische und arithmetischen Fachtexte umfassend belegt (Habermann 2001: 510-511, Gärtner 2000: 238). Das passte zum einen zur Absicht der Autoren, dem Laien mit der Fachprosa in der Volkssprache direkte Handlungsanweisungen zu geben. Zum anderen folgten die Autoren damit dem noch weitgehend mündlichen Stil des sich gerade erst formenden mittelhochdeutschen Schriftgebrauchs (Habermann 2001:58-62). Auch für die Fachprosa galt gleichwohl der humanistische Anspruch an einen guten Schreibstil, der schlicht, aber trotzdem elegant gehalten sein sollte (genus subtile). Monotonie und Wortwiederholungen sollten vermieden werden, wofür Satzvariationen und ein umfangreiches Vokabular (copia verborum) das Mittel der Wahl waren (ebd.: 225-244).

Gemeinsam mit den Koch-, Kräuter- und Volksmedizinbüchern waren die Rechenbücher eine der populären Textgattungen des frühen Buchdrucks. (Schneider 2005: 64-66). Das erste deutschsprachige Rechenbuch, das ,Bamberger Blockbuch', dass noch im Holzdruck hergestellt worden war, ist für 1482 belegt. Um 1500 kam es dann zu einem sprunghaften Anstieg bei den Neuerscheinungen (Unger 1888: 35-56, Günther 1969 [1887]: 300-308). Wie die andere Fachprosa waren die Rechenbücher, neben der Verwendung im Unterricht, auch zum Selbststudium und zum Nachschlagen vorgesehen. Widmann (1489: fol. $3^{\mathrm{r}}$ ) rühmt beispielsweise seine sorgsam ausgewählten Beispiele, „die eyn ytlicher auch mittlerer vernunfft von ym selbst wol mag versten vnnd begreyffen". Die Rechenmeister Johann Fischer und Sigmund Suevus heben in den Vorreden ihrer Rechenbücher den Vorzug des Unterrichts vor dem offenbar üblichen Selbststudium hervor (Grosse [1901] 1965: 35).

Die Rechenbücher begannen in der Regel mit einer ,Vorrede‘, in der im humanistischen Geiste der Nutzen der Rechenkunst für den ,gemeinen Mann', das heißt für den Nichtgelehrten, gepriesen wurde. Der fachliche Teil stellte am Anfang das Zahlensystem und die verschiedenen Rechenarten vor. In der ,Practica', einer umfangreichen Sammlung von Beispielaufgaben mit kaufmännischen und Handwerksbezügen, wurden dann die Rechenarten, der Dreisatz und dessen weiterführende Varianten geübt. Häufig schlossen die 
Rechenbücher mit einigen weiterführenden Rechenregeln, vor allem der regula falsi, und mit mathematischen Rätseln und Denksportaufgaben der Unterhaltungsmathematik. In den Rechenbüchern wurden die Rechenregeln vorgestellt und geübt, jedoch nicht begründet - ein wesentlicher Gegensatz zu den akademischen Werken der Mathematik. Nicht behandelt in den Rechenbüchern wurden, abgesehen vom Bruchstrich sowie dem Plus- und Minuszeichen, algebraische Notationen und Umformungen. Ebenso wenig wurden die für Kaufleute und Handwerker nicht notwendigen Gleichungen höheren Grades besprochen, obwohl viele Autoren von Rechenbüchern, darunter auch Widmann und Ries, in diesem Bereich große Kenntnisse hatten. Auch die Geometrie war in der Regel kein Bestandteil der Rechenbücher (Unger 1888: 35-62). Für die besonderen Belange der Fassmacher, die beim ,Visieren' ein Fass ausmessen und dessen Inhalt berechnen mussten, wurden jedoch oft eigene, Visierbücher' mit geometrischen Inhalten angeboten (Folkerts 2008). So wurde an einige Auflagen von Ries' zweitem Rechenbuch das Visierbuch des Frankfurter Mathematikers Erhart Helm angehängt (ebd.: 20, 30).

\section{Inhalt und Aufbau der Rechenbücher von Ries und Widmann}

Ries und Widmann standen beide hinsichtlich der Kenntnisse der Arithmetik und der Algebra auf der Höhe der Zeit. Gleichwohl bemühten sie sich, die Leser ihrer Rechenbücher nicht zu überfordern und ihnen, in Widmanns Worten, „leichte vnd nicht so geringe alß nutzpar Regelnn der Rechnung“ zu erläutern (Widmann 1489: fol. $3^{\mathrm{r}}$ ). In der Auswahl und Anordnung des Lehrstoffs gingen beide jedoch unterschiedliche Wege. Ich beginne mit Ries. Sein zweites Rechenbuch eröffnet er mit einem kurzen Vorwort und gliedert es dann, dem damals üblichen Schema folgend, in drei Teile: die Einführung in die Rechenarten, die ,Practica' und die Unterhaltungsmathematik.

Der erste Teil beginnt mit einer knappen Einführung in das indische Positionssystem mit arabischem Ziffernsystem. Ries verzichtet wie in den meisten Rechenbüchern der Zeit auf eine Beschreibung der geläufigen, aber für das schriftliche Rechnen ungeeigneten römischen Zahlen. Die Grundrechenarten Addition, Subtraktion, Duplieren, Medieren, Multiplikation und Division werden sowohl für die Linienrechnung als auch die schriftliche Rechnung mit einfachen Beispielen vorgestellt. Die Behandlung der Linienrechnung hat für Ries zum einen didaktische Gründe, wie er in der Vorrede seines dritten Rechenbuchs erläuterte: Da diese Methode kein Kopfrechnen beinhalte, übe der Schüler mit ihr zunächst den Zahlenbegriff ein, bevor er zu den Operationen des schriftlichen Rechnens komme (Deschauer 1992: 10). Zum anderen geht Ries offensichtlich auch davon aus, dass viele Kaufleute und 
Handwerker nach wie vor auf den Linien rechneten wie auch sein Titelbild belegt, welches eine typische Kontorszene zeigt (vgl. Abb. 2). Er erläutert beispielsweise bei einer der Übungsaufgaben zum Dreisatz ausdrücklich, wie bei der Linienrechnung mit dem nicht weiter teilbaren Rest einer Division umgegangen wird (Ries 1522: fol. $C^{2 v}$ ).

Beim schriftlichen Rechnen vermittelt Ries die damals gängigen Rechenund Notationsmethoden. Bei der Division lehrt er etwa die umständliche, aber allgemein übliche Notation des Überwärtsdividierens (bei dem die Reste über dem Dividend geschrieben werden). Auf eine Darstellung der einfacheren und heute gängigen, damals aber wenig verbreiteten Schreibweise des Unterwärtsdividierens verzichtet er, obwohl ihm das von italienischen Rechenmeistern bereits erwähnte Verfahren wahrscheinlich geläufig war (Unger 1888: 73-86). Als Probe werden die Umkehroperation und die oft verwendete, von ihm aber

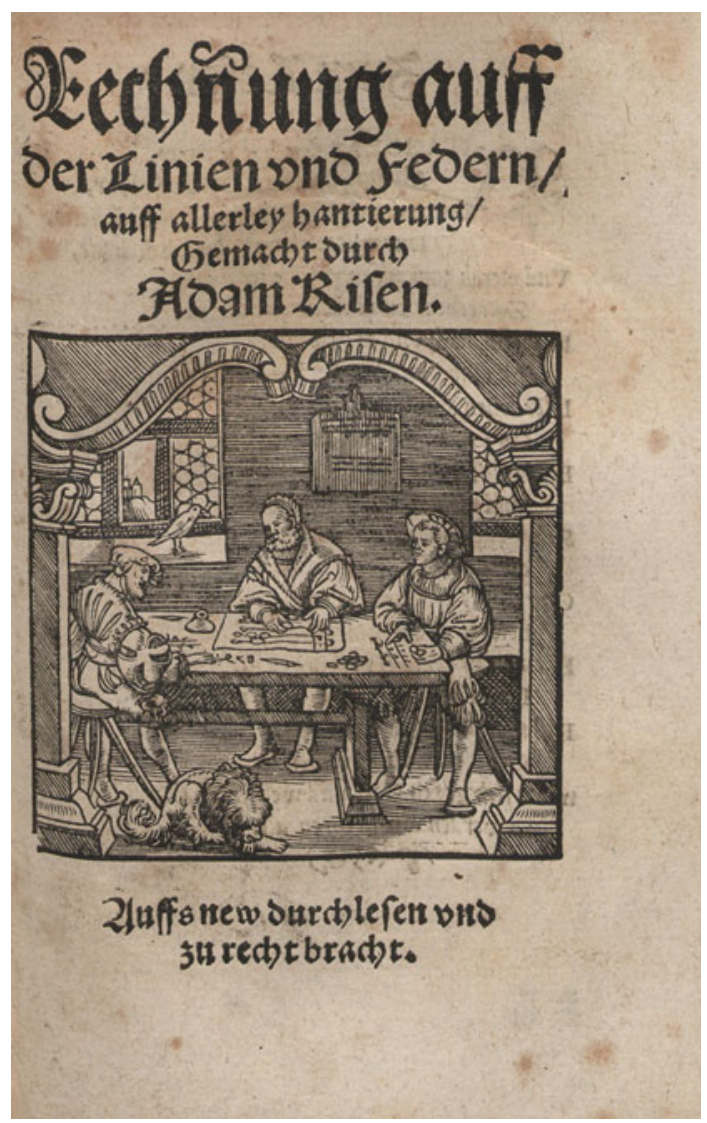

Abb. 2 Titelblatt von Ries' zweitem Rechenbuch in der Neuauflage von Michel Blum 1548 (SLUB Dresden, http://digital.slub-dresden.de/ppn273513583 [9.9.2010]) 
nur gering geschätzte Neunerprobe vorgestellt (Deschauer 1992: 132). Die ihm ebenfalls bekannte Siebenerprobe lässt er dann gleich ganz weg.

Danach werden als traditionelle Rechenarten die Summenformeln für arithmetische und geometrische Reihen eingeführt. Auf die schwierigere Berechnung quadratischer und kubischer Wurzeln verzichtet Ries dagegen mit der Begründung, dass er dies „zu seyner zeyt“ beim Berechnen des Fassinhalts und der Erläuterung algebraischer Regeln erklären würde (Ries 1522: fol. $\left.\mathrm{B}^{6 \mathrm{v}}\right)$. In seinem dritten Rechenbuch und im zu Lebzeiten unveröffentlichten Manuskript der Coß finden sich dann auch die entsprechenden Erläuterungen (Deschauer 1992: 141). Direkt anschließend, und damit anders als die meisten Autoren von Rechenbüchern (Unger 1888: 83-84), erläutert Ries den Dreisatz und rückt ihn so für den Leser beziehungsweise den Schüler in die Nähe der Grundrechenarten. Um der praktischen Bedeutung des Dreisatzes gerecht zu werden, schließt Ries - zum ersten Mal - eine Reihe von Sachaufgaben mit Lösungen an und geht auch auf den Umgang mit den Stückelungen von Geld- und Wareneinheiten ein. Für den Fall, dass bei der Division im Dreisatz ein Rest bleibt, führt er den euklidischen Algorithmus für die Bestimmung des größten gemeinsamen Teilers ein, um den entstandenen Bruch so weit wie möglich zu kürzen.

Erst nach dieser Motivation werden die gebrochenen Zahlen im Allgemeinen vorgestellt. Den Wert eines Bruchs („wie viel ein jeglicher Bruch in sich behelt") veranschaulicht er am Beispiel von Währungen, Gewichten und anderen Einheiten, indem er den Zähler in die nächstkleinere Einheit auflöst und dann zu einem ganzzahligen Wert gelangt (Ries 1522: fol. $C^{3 v}$ ). Darauf schließen sich Erläuterungen zu den Grundrechenarten für Brüche und zum Berechnen der Bruchteile von Brüchen an. Wie bisher besteht die Erklärung aus einer kurzen allgemeinen Beschreibung des Rechenwegs und einigen wenigen Zahlenbeispielen. Danach folgen um die dreißig Sachaufgaben in steigendem Schwierigkeitsgrad. Die meisten Aufgaben behandeln den Dreisatz mit gebrochenen Zahlen. Am Ende des Abschnitts werden dann jedoch komplexere Aufgaben gestellt, die sich nur teilweise mit dem Dreisatz lösen lassen. Offensichtlich geht es Ries an dieser Stelle darum, den Schüler nach dem Üben des Dreisatzes zu verdeutlichen, dass nicht alle Rechenaufgaben mit der regula de tri allein gelöst werden können.

Auf Basis der bisherigen Rechenregeln stellt Ries im zweiten Teil des Rechenbuchs eine große Folge von Anwendungsaufgaben, die er thematisch nach dem Bedarf der Kaufleute und einiger rechenintensiver Gewerke aus dem Bergbau und der Münze gliedert: Goldwährung, Geldwechsel, Silber- und Goldrechnung, Beschickung des Schmelztiegels, Münzschlag, Gewinnverteilungen in Gesellschaften und Warentausch. Dabei werden als Varianten des Dreisatzes die Zinsrechnung, die Mischrechnungen für Legierungen und die Verhältnisrechnung für Gesellschaften eingeführt. Alle Aufgaben geben praktische Fragestellungen wieder. In den einzelnen Abschnitten wird der 
Schwierigkeitsgrad der Aufgaben jeweils gesteigert. Ries gibt bei jeder Aufgabe die Lösung an und beschreibt den grundsätzlichen Rechenweg, ohne ihn aber im Detail vorzuführen. Bei schwierigen Aufgaben gibt er auch Proberechnungen an.

Nach der ,Practica' folgt der dritte und letzte Abschnitt zur Unterhaltungsmathematik. Dazu führt Ries als zweite Rechenregel neben dem Dreisatz die regula falsi ein. Anders als bis dahin hat der überwiegende Teil der Beispielaufgaben nun keinen praktischen Bezug mehr, so etwa gleich die erste Aufgabe:

Item eyner spricht got grueß euch gesellen all 30 antwurt eyner wan unser noch souil // und halb souil wern // so wern unnser 30.die frage wieuil yr gewesen? (Ries 1522: fol. $\left.\mathrm{G}^{7 \mathrm{v}}\right)^{3}$

Auch die Beispiele, die in einen Handelsbezug eingebettet sind, haben kaum direkten Bezug zur rechnerischen Praxis eines Kaufmanns:

Item / eyner hat 20 pfundt Saffran unnd Ingwer durcheinander-kost ein pfunt saffran 3 flo: [Florin, Gulden] / und 2 pfunt Ingwer ein flo: [ver]kaufft darauß 45 floren- Nun frage ich / wievil yetzliches pfundt in sonderheyt gewesen seyn? (ebd.: fol. $H^{6 v}$ f.)

Der letze Abschnitt des Rechenbuchs soll den Schüler vor allem befähigen, bekannte Aufgaben der Unterhaltungsmathematik selbst zu lösen. Eine unmittelbare praktische Bedeutung hat dieser für den angehenden Kaufmann oder Handwerker nicht. Gleichwohl sollen aber anscheinend fortgeschrittene Schüler angeregt werden, auch die regula falsi als mögliche Rechenregel für die Praxis in Betracht zu ziehen. Darauf deuten die in einen Handelsbezug eingebetteten Aufgaben zum doppelten falschen Ansatz hin, die zum Teil frühere Aufgabenstellungen aufgreifen und sich auch mit dem Dreisatz berechnen lassen (Deschauer 1992: 180-181). Bei den abschließenden Aufgaben tritt dann der Unterhaltungszweck wieder ganz in den Vordergrund. Ries stellt in einigen Aufgaben die Zechrechnung ${ }^{4}$ vor, beschreibt die Konstruktion magischer Quadrate und schließt sein Buch mit einer Aufgabe der Bewegungsrechung. ${ }^{5}$

Widmanns Behende vnd hubsche Rechenung ist inhaltlich viel umfassender als Ries' Rechenbuch angelegt und beginnt mit einem langen Vorwort sowie einer umfangreichen Inhaltsangabe. Bei der Vorstellung der Rechenarten im ersten Abschnitt behandelt Widmann auch das Ziehen der quadratischen und kubischen Wurzeln, die in der Rechenwelt des Kaufmanns und Handwerkers nicht vorkommen. Er führt zudem die traditionelle, aber umständliche Tolletrechnung für die Berechnung von Preisen bei mehrsortigen Geld- oder Wareneinheiten ein, bei der zunächst in Hilfstabellen das Zehn-, Hundert-, Tausendfache und so weiter des Preises je Wareneinheit notiert wird, um dann daraus den Preis für die tatsächliche Warenmenge zu bestimmen. Ries und die meisten anderen Rechenmeister bleiben bei solchen 
Aufgaben dagegen beim einfacheren Dreisatz (Unger 1888: 94-95, Tropfke 1980: 365).

Im zweiten Abschnitt stellt Widmann zunächst das euklidische Rechnen mit Proportionen vor, das für den Kaufmann und Handwerker keinerlei praktische Bedeutung hatte. Anschließend führt er den Dreisatz und eine Vielzahl von Rechenregeln ein, darunter auch die von Ries behandelte regula falsi. Die Gliederung des Textes erfolgt nach den lateinischen Bezeichnungen der Rechenregeln. Teils übernimmt Widmann traditionelle Namen für die Regeln, teils führt er selbst Bezeichnungen für sie ein. Die Rechenbeispiele zu den Regeln fasst er zum Teil in kaufmännische und handwerkliche Bezüge ein und stellt sie unter entsprechende Unterschriften. Eine Vielzahl der Regeln und Aufgaben haben jedoch keinen direkten praktischen Nutzen für den kaufmännischen Rechner, sondern widmen sich allgemeinen algebraischen Fragestellungen (Kaunzner 1968: 74-81). Beispielsweise behandelt Widmann mit der vierten regula pulchra (= schöne Regel) in heutiger Schreibweise die Lösung der Gleichungen $x+a=b(y-a)$ und $y+a=c(x-a)$ und stellt dann folgende Übungsaufgabe:

Gelt

Itm eß kumpt eyner zu dem Anderenn Und spricht ich hab szo uil gelcz [Geld].vnd wen du mir deyner d [Pfennig] 1 dar zu gebst szo het ich zwey mol als vil als du Spricht der ander zu ersten Ich hab szo vil gelcz Und wenn du mir deynrs gelcz $1 \mathrm{~d}$ dar zu gebst so het ich wol dreymol als vil als du. $\mathrm{Nu}$ ist die frag wie vil hat itlicher gelcz gehapt (Widmann 1489: fol. 121 $1^{\mathrm{r}}$ )

Anschließend führt Widmann den Leser im dritten Abschnitt in zwei- und dreidimensionale geometrische Formen und in die Berechnungen verschiedener Strecken, Umfänge und Inhalte ein. Diese Inhalte hatten aber für den Kaufmann und für die allermeisten Gewerke in der Praxis keine Bedeutung. Wie Ries schließt er das Rechenbuch mit einigen wenigen Aufgaben der Unterhaltungsmathematik, namentlich der Zech- und der Bewegungsrechnung.

In der Gegenüberstellung von Ries' und Widmanns Rechenbüchern wird der für den Kaufmann und Handwerker besser geeignete Inhalt und Aufbau der Rechenung auff der Linien unnd Federn deutlich. Ries wägt genau ab zwischen der Praxistauglichkeit seines Rechenbuchs einerseits und der Behandlung des erwarteten traditionellen Kanons der Rechenkunst andererseits. Anders als Widmann und viele andere Rechenbuchautoren behandelt er sowohl das Linien- als auch das schriftliche Rechnen. Von den traditionellen Rechenarten, die für sein Publikum keinen praktischen Nutzen haben, behält er nur die leichten bei, die schwierigen, wie das Wurzelziehen, streicht er. Ähnlich souverän ist die Behandlung der regula detri: Auch wenn es der traditionellen Ordnung der Rechenarten in den meisten deutschen Rechenbüchern widerspricht, behandelt er den für die Rechenpraxis äußerst wichtigen Dreisatz direkt bei der Vorstellung der anderen Rechenarten. 
Seine ,Practica' gliedert Ries nach Anwendungsbereichen, die dem Bedarf der Kaufleute und der in Süddeutschland wichtigen Gewerke Bergbau und Münze entsprechen. Der Zugang für den Schüler und Leser ist damit auf die praktische Regelanwendung hin orientiert. Die ebenfalls traditionelle Unterhaltungsmathematik ist dann für den Leser deutlich sichtbar von der ,Practica‘ abgegrenzt.

Der Inhalt von Widmanns Rechenbuch ist dagegen sichtlich umfangreicher und für den Alltagsbedarf des kaufmännischen Schülers schwerer zu erschließen: Die vorgestellte Mathematik übersteigt mit Proportionenrechnung, Geometrie und allgemeinen algebraischen Problemen deutlich den Bedarf der Kaufleute und Handwerker. In der ,Practica' ist weder aus der Anordnung noch aus den Bezeichnungen der Rechenregeln und Aufgaben erkennbar, wo es sich um Inhalte für die kaufmännische Praxis handelt und an welchen Stellen der abgehandelte Stoff über diesen Bedarf hinausgeht. Der praktische Nutzen von Behende und hvbsche Rechenung war damit für den angehenden Kaufmann und Handwerker deutlich geringer als bei Ries' Rechenbuch.

\section{Die Gestaltung der Erklärungen und Übungsaufgaben}

Ries' und Widmanns Rechenbücher hatten trotz ihrer unterschiedlichen Inhalte gleichermaßen den Anspruch, Lehrbücher für den Schul- und den Selbstunterricht zu sein. Die beiden Hauptbestandteile des Rechenunterrichts und damit auch der Rechenbücher bildeten die Erläuterung von Rechenarten und -regeln sowie die Durchführung entsprechender Übungsaufgaben (Gärtner 2000: 236-237). Die didaktische Ausgestaltung dieser beiden Typen von Teiltexten bei Ries und Widmann wollen wir nun an zwei Beispielen näher betrachten.

Ein gut geeignetes Beispiel für eine Regelerläuterung ist der für das kaufmännische Rechnen unabdingbare Dreisatz: eine vergleichsweise einfache Rechenregel, die aber nicht zum Lehrkanon des Mathematikunterrichts in den auch von Kaufmanns- und Handwerkerkindern besuchten Kirch- und Lateinschulen gehörte und daher einer besonders guten Erläuterung bedurfte. Generell halten die Erläuterungen von Rechenarten und -regeln in den Rechenbüchern von Ries, Widmann und anderen Autoren ein festes Schema ein. Nach einer kurzen Definition folgen eine allgemeine Erklärung der Rechenart oder -regel, ein oder mehrere einfache Rechenbeispiele und häufig noch die Probe. Anschließend schließen sich meist mehrere Beispielaufgaben mit Lösungen an (ebd.: 237). Im Rahmen dieses Ablaufs zeigen sich bei Ries und Widmann deutliche Unterschiede. Beginnen wir mit Ries' Behandlung des Dreisatzes: 


\section{Regula Detri}

Ist ein regel von dreyen dingen / setz hinden das du wissen wilt / wirt die frag geheysen // das ym vnter den anderen zweyen am namen gleych ist.setze forn / vnd das einander dingk bedeut mitten.Darnach multiplicir das hinden vnd mitten stet durcheinander / das darauß koemet.teyl ab mit dem fordern / so hastu wie theur das drit koemet vnnd das selbig ist am namen gleych dem mitteln als hie in volgendem exempel

Item 32 eln tuchs für 28 flo: wie komen 6 eln facit 5 flo: 5 groschen 3 pfennig setz also: eln 3228 flo: 6 eln (Ries 1522: fol. $\mathrm{B}^{6 \mathrm{v}}$ )

Ries verzichtet vollständig darauf, die Bedeutung des Dreisatzes hervorzuheben. Der äußerst knappen Einführung des Dreisatzes als der „regel von dreyen dingen" folgt eine ebenfalls sehr kompakte Erläuterung des Verfahrens. ${ }^{6}$ Definition und Erläuterung sind für sich genommen sehr kurz gehalten und kaum verständlich, da sie, wie in allen Rechenbüchern der Zeit, rein sprachlich formuliert sind. In Zusammenhang mit dem Beispiel wird das Gesagte aber direkt einsichtig. Zur Nachvollziehbarkeit trägt insbesondere das Notationsschema der drei bekannten Werte (32 -28 - 6) bei, das Ries von nun an in den meisten Dreisatzaufgaben verwendet. Die enge Verbindung von theoretischer Erklärung und praktischer Übung, die einander bedingen, machen den hohen didaktischen Nutzen dieser Einführung aus: Die Erklärung wird verständlich, indem der Schüler das vorgestellte Schema sogleich am Beispiel anwendet. Die im Vergleich zu Widmann und anderen Autoren hervorstechende Kürze von Definition und Erläuterung ist keine Schwäche, sondern vielmehr wesentlich für ihre Wirkung. Definition, Erläuterung und Beispiel können und sollen als Einheit in einem Zuge vom Lehrer vorgelesen und vorgerechnet oder vom Schüler selbst gelesen werden. Zu dieser didaktisch bedingten Verkürzung gehört auch, dass Ries den Dreisatz als Lösung für Aufgaben mit PreisWarenmengen-Beziehungen einführt („so hastu wie theur das drit koemet"). Die Vorstellung der Probe beendet die Erläuterung des Dreisatzes. Die direkt folgenden Übungsaufgaben beziehen sich zunächst durchgehend auf die häufigste Aufgabenstellung in der Praxis: die Ware-Preis-Relation. Erst in späteren Aufgaben, wenn Ries davon ausgehen kann, dass sich das Schema beim Schüler verfestigt hat, verwendet er den Dreisatz auch für andere Relationen.

Im Aufbau ähnlich, aber in längerer Form und mit einem Währungstausch als Eingangsbeispiel behandelt Widmann den Dreisatz. Nachdem er ausführlich dargelegt hat, wofür der Kaufmann das Rechnen und insbesondere den Dreisatz benötigt, nämlich um nicht betrogen $\mathrm{zu}$ werden, folgt die eigentliche Erläuterung:

Unnd ynn dem wil ich dich zum ersten lernen durch die gulden Regel die dan alszo genant ist wan gleicher weysz als das golt vbertrit all ander metal also auch diesze Regel in gebrauchung vbertrit al ander Regel Auch wirt sy genat regula Detri wan in yr durch drey bekante zal wirt die viert vnd vnbekant gefunden Sy ist auch recht genant Regula proportionum Wan in der regel werden erkant vnd erfunden alle 
proportiones als dan klerlich ausz drucket Euclides in dem sybenten vnd auch sechsten buch vnd ander mer Nu aber diesze wort kurzlichen ab zuschneiden Soltu wyssen alsz vnsz hie her dient das Regula Detri (die dan hubscher eygenschaft zwelf an yr hat hie her nicht zu verzelen) nicht anders ist (als vnsz dan die meyster der freyen kunst sagen) dan drey dingk die du seczt vnter welichen das erst vnd das lezte almol musz gleich sein Weliches lezte du solt multipliciren mit dem mittelsten das dann gleich ist dem vierden vnd unbekanten.dz erwechst auß solcher multiplicatio- vnd der teylung daß product mit dem ersten. vnd also soltu albeg das selbige unbekant $\mathrm{dz}$ da du dan wissen wilt vnd darnach die frage ist. hinden seczen vnd mit dem ersten multipliciren Und darnach das erwachsen product durch das erste teylen.vnd dan ausz solcher teylung kumpt das ist die vierde vnd unbekante zal gewesen vnd bericht die frag Alsz yn dieszem nachgeenden auff diesze Regel geordinirten exempeln gancz klerlichen wird auszgedruckt vnnd zvm Ersten ein exemel von ganczer zal also / 100 ducl [Dukaten] pro 129 floren guldn wy kummen 34 ducl Machs also nach der regel-multiplicir da leczt mit dem mitelsten-als 34 mit 129 ader widerumb und kumpt 4386. Das teil in das erst.vnd kumpt 43 floren 17 ß [Groschen] 2 hel. [Heller] 2/5 vnnd ist recht. (Widmann 1489: fol. 75r f.) ${ }^{7}$

Widmanns Definition, die noch einmal auf die praktische Bedeutung des Dreisatzes und zusätzlich auf den Bezug zu Euklids Proportionenlehre verweist, ist deutlich länger als die von Ries. Erläuterung und Beispiel sind inhaltlich gleich zu Ries, jedoch, wie die Definition, umständlicher formuliert und länger. Anders als Ries führt Widmann den Dreisatz zudem nicht als Regel für dessen häufigste Anwendung, die Preis-Waren-Relation, sondern gleich in allgemeiner Form ein. Auch sein Rechenbeispiel behandelt nicht den gängigen Fall, den Preis einer Ware zu bestimmen, sondern den Umtausch von zwei Währungen. Er folgt damit der Tradition der libri d'abbaco, die häufig, wenn auch nicht immer, mit einem Beispiel zum Geldtausch beginnen (Høyrup 2007: 62-63). Anders als ihre italienischen Kollegen beschäftigten sich jedoch die meisten deutschen Kaufleute weniger mit reinen Geldgeschäften, sondern vorrangig mit dem Tausch von Ware gegen Geld. Widmanns Einführungsbeispiel geht damit, anders als bei Ries, an ihrem vorrangigen Einsatz des Dreisatzes vorbei. In noch stärkerem Maße gilt dies für die Handwerker unter Widmanns Lesern.

Die Übungsaufgaben zum Dreisatz folgen erst nach zwei längeren Abschnitten zu den konkreten Stückelungen verschiedener Münzen und Gewichte sowie der Ankündigung von zwei Rechenregeln für die Bestimmung unbekannter Unterteilungen von Waren- und Geldeinheiten. Insgesamt verliert Widmanns Erklärung des Dreisatzes gegenüber Ries an Prägnanz. Weder bilden Definition, Erläuterung und Beispiel eine beim Lesen oder Vorlesen erfassbare Einheit noch bezieht sich die Erklärung direkt auf die gängige Anwendung des Dreisatzes: die Preisberechnung für eine Ware.

Widmanns Neigung zur ausführlichen und umfassenden Erklärung, die den Schüler eher überfordert als ihm hilft, ist nicht auf den Dreisatz beschränkt. Sie zeigt sich in ähnlicher Form etwa auch bei seiner Behandlung der Grundrechenarten: Als Proben stellt er neben der Umkehroperation und der 
Neunerprobe auch die Siebenerprobe vor, ohne die besonderen Vorteile der letzten zu erläutern, nämlich ihre höhere Zuverlässigkeit. Widmann steht mit seinem Hang zur Ausführlichkeit und Vollständigkeit nicht allein. In der Definition des Dreisatzes ist in den meisten Rechenbüchern eine ausführliche Begründung seiner Bedeutung üblich (Unger 1888: 86). Die Rechenmeister Christoph Rudolff, Peter Apian und Johann Fischer führen neben der Neunerund der Siebenerprobe auch noch die Sechser- und die Achterprobe ein (ebd.: 83).

Auch bei den Rechenaufgaben zeigen sich ähnliche Unterschiede zwischen Ries' und Widmanns Darstellungen. Ein geeignetes Beispiel dafür ist die Zinseszinsrechnung: eine wichtige, aber aufwändige Rechnung, bei der es vor allen darauf ankommt, Rechenvorteile zu nutzen. Ries gibt hierfür eine einzige Beispielaufgabe an. Anhand der Angabe von Kapital, Zins und Laufzeit soll die Schuld nach mehreren Zinsperioden berechnet werden:

Item ein Jud leyhet eynem 20 flo: 4 Jar vnd alle halbe Jar rechent er den gewin [Zins] zum hauptgut [Kapital] Nun frage ich wieuil die 20 flo: angezeigte 4 Jahr bringen muegen: so alle wochenn 2 pfen: von einem flo: gegeben werden. facit gewin vnd gewins gewin / etc. 69 fl:14 gro: / 9 pfen: vnd 2123648028045/3938980639167 teyl. Machs also rechen zum ersten wieuil die 20 flo: ein halb iar tragen sprich ein wochen gibt 40 pfen: was geben 26 wochen? facit 1040 pfen: nun mach die 20 flo: zu pfen: komen 5040 pfen: hauptgut addir den gewinn / komen 6080 sprich. 5040 pfen: geben 6080 Das erste halbe iar / was geben 6080 Das ander halb iar Das 0 lesche forn vnd mitten auß / also:

\section{0}

Die forderste vnd mittelste zal laß gegen einand auffgehen dan es wurde sunst zcu hoch steygen im multiplicirn stet

\section{0}

Multiplicir / vnnd teyl nicht ab: sundern schreyb den teyler darunder / koemet hauptgut vnd gewin Das ander halbe Jahr darnach sprich aber 63 geben 76. was gibt hauptgut und gewin zusamen auß dem andern halben iar komen machs also hynfort biß vff die acht halbe iar: darnach diudir mit dem teyler / welcher dir komen wirt so hastu eitel [nur] pfen: die mach zcu grosch: vnnd als dan groschen zu floren // so koemet das facit / wie oben vorzeychent. (Ries 1522: fol. F.)

Ries folgt hier, wie bei fast allen seinen Rechenbeispielen, dem Ablauf Aufgabenbeschreibung, Frage, Lösung und gegebenenfalls Hinweise zum Rechenweg. Mit der Angabe der Lösung direkt nach der Frage weicht Ries vom üblichen Schema der libri d'abbaco und der meisten deutschen Rechenbücher ab, das die Lösung an das Ende der Übungsaufgabe stellt (van Egmond 1980: 16-17, Gärtner 2000: 237). In seinem Aufbau der Rechenbeispiele konnte der fortgeschrittene Schüler gleich beginnen, die Aufgabe selbst zu rechnen und dann mit der angegebenen Lösung zu vergleichen, ohne noch den Text zum Rechenweg studieren zu müssen.

Bei einfachen oder schon mehrfach geprobten Aufgaben nennt Ries überhaupt keinen oder nur einen verkürzten Lösungsweg, oder er beschränkt sich darauf, den Ansatz des Dreisatzes anzugeben. Ein typisches Beispiel für einen verkürzten Rechenweg bei Ries ist folgende Lösung einer 
Dreisatzaufgabe: „Mach's so: Löse 1 Gulden in Pfennige auf; es kommen 251 Pfennig heraus. Dann multipliziere die übrigen Gulden, teile und wandle in Pfunde um." (Übertragung ins Hochdeutsche: Deschauer 1992: 61). In unserem Beispiel bietet er jedoch, wie auch sonst bei anspruchsvollen Aufgaben, mehr Hilfestellungen. Zunächst sollen alle Beträge in Pfennige umgerechnet werden. Über den Dreisatz berechnet er, dass nach einem halben Jahr aus 5040 Pfennigen mit dem Zins 6080 Pfennige geworden sind. Dieses Verhältnis nimmt er zugleich als Vermehrungsrate für alle weiteren halbjährlichen Zinsrechnungen nach dem Dreisatz, kürzt diese Rate aber zunächst rechenfreundlich um 10 und dann um 8 zu 76 : 63. Zusätzlich empfiehlt er, bei der Berechnung des Zinseszinses nach acht Perioden die Divisionen bis zum Ende aufzuheben und vermeidet damit den für sein Publikum schwierigen Umgang mit Bruchzahlen so lange wie möglich.

Widmann führt die Zinseszinsrechung ähnlich vor, unterscheidet sich aber in der methodischen Darstellung deutlich von Ries:

\section{Gwin und hauptgut}

Item eyner hat in eyn wechselpanck gelegt $1000 \mathrm{fl}$ vnd der wechseler sol ym eynß yden Jars $4 \mathrm{fl}$ von 100 geben. vnd das ligt also 4 Jar $\mathrm{Nu}$ ist die frag wye vil das hauptgut vn gewinß gewin die 4 Jar gwint Und wie vil es in eyner sum mach Wiltu das wissen vnd des gleichen Szo machß also Secz mol also 104/100 104/100 104/ 100 104/100 Und dz darumb das 100 die vnten sten 104 weren eyn Jar Darumb multiplicir auch die oberen [sic! Muss , die unteren' heißen] alle zusammen vnd kumpt [kommt] 100000000 Und also mltiplicir auch die oberen alzo zusammen vnd kummpt 116985856 vnnd secz darnach auff die Regel Detri alszo vnd sprich $100000000 \mathrm{fl}$ geben $116985856 \mathrm{wz}$ [was] geben $1000 \mathrm{fl}$ vnd kumpt $1169 \mathrm{fl}$ 2638/ 3125 vnd szo vil wirt die 4 Jar auß $1000 \mathrm{fl}$ (Widmann 1489: fol. $130^{\mathrm{r}}$ f.)

Widmanns Aufgabe ist einfacher gehalten als die von Ries, da er nur mit Dukaten rechnet. Die Rechenvereinfachung des Resolvierens in die kleinste Währungseinheit führt er daher nicht vor. Auch in den anderen Aufgaben der ,Practica' verzichtet Widmann in der Regel auf das wichtige, aber schwierige Rechnen mit gestückelten Währungseinheiten, die bei Ries einen großen Raum einnimmt. Sein Rechenweg ist dann prinzipiell der gleiche wie bei Ries. Mit der Notation der vier Zinsverhältnisse 104 : 100 stellt Widmann den Weg sogar noch deutlicher dar. Unglücklicherweise wählt er seine Zahlen aber so, dass das weitere Kürzen dieses Verhältnisses nicht zweckmäßig erscheint. Anders als Ries verzichtet Widmann daher auf die Vorführung des Kürzens bei der Multiplikation der Zinsverhältnisse. Bei der Durchführung der Zinseszinsrechnung nach Ries' und Widmanns Methode ist dieses frühzeitige Kürzen jedoch sehr wichtig, weil die folgenden Multiplikationen und die abschließende Division wegen der großen Zahlenwerte sonst unnötig erschwert werden. Generell ist Widmanns Vorführung der Lösungswege schematischer als Ries. Alle Rechenansätze der ,Practica' werden mit der Angabe aller Zahlenwerte und Zwischenergebnisse, aber ohne mögliche Vereinfachungen, vorgestellt. Zudem nennt Widmann die Lösung einer 
Aufgabe fast immer erst am Ende und steht damit in der Tradition der libri d'abbaco und der meisten deutschen Autoren von Rechenbüchern. Der geübte Schüler, der lediglich eine Textaufgabe und das Ergebnis benötigt, wird so in seinem Lesefluss behindert.

Insgesamt überwiegen bei der Analyse der einzelnen Erläuterungen und Rechenbeispiele zunächst die Gemeinsamkeiten zwischen Ries und Widmann. Beide folgen dem dogmatischen Schema der zeitgenössischen Rechenbücher. Ries legt jedoch deutlich mehr Wert auf kurze Erläuterungen, in denen Definition, Erläuterung und Beispiel als Einheit gelesen und verstanden werden können. Bei Widmann und vielen anderen Autoren gehen die Zusammenhänge häufig in zu ausführlichen Darstellungen verloren. Bei den Rechenaufgaben nennt Ries die Lösung, anders als Widmann und die anderen Autoren von Rechenbüchern, fast immer direkt nach der Aufgabenstellung und macht sie so für den selbstständig übenden Leser leichter auffindbar. Seine Beschreibungen des Lösungswegs sind flexibler als die von Widmann und nehmen mehr Rücksicht auf ihren Kontext im Buch: Bekanntes wird nicht mehr oder nur verkürzt vorgeführt, Neues dagegen ausführlich erklärt. Ries' kompakte und kontextbezogene Darstellungen waren vor allem für das Selbststudium sehr hilfreich. Ohne Lehrer war der Leser besonders auf eine differenzierte Aufbereitung des Lehrstoffs angewiesen.

\section{Sprache, Fachvokabular und Notationsschemata}

Die deutschsprachigen Rechenbücher des 15. und 16. Jahrhunderts folgten in ihrem Sprachstil dem Vorbild der libri d'abbaco und anderer Textarten der deutschsprachigen Fachprosa. Kennzeichnend war dabei die direkte, imperative Ansprache des Lesers in der zweiten Person Singular und die damit einhergehende grammatikalische Beschränkung auf einfache Satzkonstruktionen. Damit wurde im Rechenbuch, wie auch in der übrigen Fachprosa der Zeit, die übliche mündliche Vermittlung des Lehrstoffs nachgeahmt, hier der dogmatische Vortrag des Rechenmeisters. Auf die Verwendung algebraischer - im damaligen Sprachgebrauch cossistischer - Notationen für Regeln und Rechnungen wurde noch weitgehend verzichtet. Es wurde jedoch bereits ein Fachwortschatz eingeführt, der im Allgemeinen noch mehrere gleichbedeutende, deutsche und lateinische Begriffe beinhaltete (Gärtner 2000: 237-239, Eichler 1992) und damit der Vorgabe des genus subtile folgte. Für die schriftlichen Rechenarten und für schwierigere Rechenregeln wurden Notationsschemata angegeben, die sich bei den Grundrechenarten fast unverändert bis heute gehalten haben. Im letzten Abschnitt soll nun verglichen werden, wie Ries und Widmann sich im Rahmen dieser stilistischen Konventionen bewegten. 
Ries folgt konsequent dem reduzierten Grammatikstil, wie ein Beispiel aus der ,Practica' verdeutlicht:

Saffran

Item ein stumpff saffran / wigt 48 pfundt: 13 lot / 3 quint: [Untereinheit des Lots] und kost ein pfundt 3 flo: / 9 ß [Schilling] / 6 hel: facit 168 flo: / 5 ß / 10 heller und 23/64: Setz ein pfundt fur 3 flo: 9 ß 6 heller. Wie komen 48 pundt / 13 lot / 3 quint. mach forn und hinden quent: darnach mitten heller. stet 128834 hel: 6199 (Ries 1522: fol. $\mathrm{D}^{4 v}$ )

Ries' Satzbau ist, wie im Beispiel, über das gesamte Buch hinweg einfach gehalten und besteht in der Regel aus Hauptsätzen, die bei Bedarf durch einfache konditionale und Objektnebensätze ergänzt werden. Die Bestandteile der Definitionen und Aufgaben werden durchgehend durch deutsche und lateinische Signalwörter gekennzeichnet. Die Erläuterungen der Rechenarten werden durchweg mit ,lernt' und ,heyßt' begonnen: „Addirn ader Summirn. Lernt wie man vil. und mancherley zaln. vonn golt / groschen / pfennig. und helleren. in eyne summa brengen sol“ (ebd.: fol. $\mathrm{A}^{3 \mathrm{v}}$ ) oder „Subtrahirn. heyßt abzihen. lert wie mann eyne zal vonn der anderen nemē sal“" (ebd.: fol $A^{4 v}$ ). In den Rechenaufgaben kennzeichnen fast durchgehend die lateinischen Signalwörter ,Item' den Beginn einer Aufgabe und ,Facit' das Ergebnis. Mit der Verwendung dieser lateinischen Begriffe setzt Ries eine Tradition älterer Fachtexte zur Arithmetik aus Deutschland fort. Sowohl in den lateinischen als auch in den deutschen Passagen der Practica des Algorismus Ratisbonensis, der Mitte des 15. Jahrhunderts im Benedektinerklosters St. Emmeran in Regensburg entstanden war, finden sich beide Begriffe (Vogel 1954). In der Abschrift einer Vorlesung aus den Jahren 1467/68 des Erfurter Mathematikers Gottfried Wolack wird das ,Item' ebenfalls verwendet, das Ergebnis dann jedoch mit dem lateinischen ,proueniunt' notiert (Wappler 1900). Beginnend mit dem Bamberger Blockbuch aus dem Jahr 1482 (Vogel 1980) werden ,Item' und ,Facit' zu oft verwandten Signalwörtern in den Rechenbüchern.

Die Erläuterung des Rechenwegs beginnt Ries in der Regel direkt mit dem imperativen Verb, oft mit den stereotypen Signalwörtern ,Machs also', ,Nimm also' oder ,Setz also'. Der Ansatz des Dreisatzes wird durchgehend entweder mit der Wiederholung der bekannten Relation und der Frage nach dem zu berechnenden Wert ('Wie komen...') oder der Liniennotation der drei bekannten Werte beschrieben ('Stet also... '). Diese stereotype Grammatik und Wortwahl soll dem Schüler helfen, sich im Text schnell zu orientieren und die Funktion eines Teiltextes sofort zu erkennen (Gärtner 2000: 213-215).

Für die Grundrechenarten und die Ansätze bei Dreisatz, Kettensatz, Mischungsrechnung, Gesellschaftsrechnung, regula falsi und Zechrechnung gibt Ries einheitliche Notationsschemata für die Anordnung der Zahlen an, die die Durchführung der Rechnung vereinfachen. Einziges mathematisches Symbol ist der damals bereits übliche Bruchstrich der gebrochenen Zahlen. In der Notation der regula falsi werden die Bezeichnungen ,plus' und ,minus' (für 
nach oben oder unten abweichende ,Lügen') verwendet, die Symbolzeichen ,+ und ,-' werden lediglich in der Regelerläuterung vorgestellt. In der Ausgabe von 1525 werden dann aber auch in den Notationsschemata der regula falsi die Symbolzeichen statt der ausgeschriebenen Rechenoperationen eingesetzt (Deschauer (1991 [1522]: 13).

Bei den Grundrechenarten verwendet Ries in der Regel einige wenige, gleichbedeutende Bezeichnungen. Dabei greift er sowohl auf die tradierten lateinischen Bezeichnungen als auch auf deren deutsche Entsprechungen zurück. Für die Division finden etwa sowohl ,dividir‘ als auch ,brichs' und ,teil ab' Verwendung. Ries beschränkt jedoch das neue Vokabular. Nicht unbedingt notwendige Fachbegriffe werden vermieden und umschrieben, etwa indem er die Verwendung des schon bekannten Fachausdrucks der ,Differenz' durch die Umschreibung ,das pleibent' umgeht (ebd.: 25-34).

Widmanns Satzbau ist in den Erläuterungen und Übungsaufgaben ähnlich einfach gehalten wie bei Ries. Sein Gebrauch von stereotypen Wendungen und Signalwörtern ist jedoch weniger streng als bei Ries, wie am Beispiel der Begriffsdefinitionen für Addition und Subtraktion deutlich wird: „Additio. $\mathrm{Nu}$ soltu wisszen das addiren heyßt zcu samen geben eyn zal zu der anderen das ein sum darauß werde. [...] Subtrahiren Hye nach wil ich dich lernen subtrahiren das heyßt ab zihen. [...]" (Widmann 1489: fol. $\left.9^{\mathrm{r}}, 11^{\mathrm{v}}\right)$. Während auch Widmann jede Rechenaufgabe mit dem Signalwort ,Item' einleitet, ist seine Angabe des Ergebnisses bei längeren Aufgaben in die Erläuterung der Berechnung mit eingebunden. Satzaufbau, Wortwahl und Verwendung von Signalwörtern sind vielfältiger als bei Ries, wie die Verkündung des Ergebnisses in vier aufeinander folgende PfefferAufgaben beispielhaft zeigen:

[...] machs nach der Regel szo kummen $91 \mathrm{fl} 18$ ß 8 hlr $1 / 4$ etc [...] Machs nach der Regel so kummen $6578 \mathrm{lb}$ [Pfund] 18/19 eynß lb und szo vil ist des pfeffers gewegen etc

[...] Machß vnd secz nach der Regel alszo 103 duc 3/4 [Dukaten] geben 1 kargk [venez. Gewichtseinheit] was geben 2430 duc vnnd kummen 23 kargk 168 lb 56/83 eynß lb vnd solt wyssen das 1 kargk ist $400 \mathrm{lb}$ zu venedig etc

[...] $\mathrm{Nu}$ addirß als zusammen alß $18 \mathrm{fl} 44 \mathrm{~B} 11 \mathrm{hlr}$ vnd 1 hlr kut $20 \mathrm{fl} 1 / 4$ vnd ist recht Also magstu alle andere prob der gleich gebrauchen. (ebd.: fol. $88^{\mathrm{v}}-90^{\mathrm{v}}$ )

Auch bei der Verwendung von Symbolen und Anordungsschemata ist Widmann variabler als Ries. An mehreren Stellen verwendet er die in der Coß zwar bereits bekannte Schreibweise mit Plus- und Minuszeichen in Termen, die hier aber unvermittelt und das erste Mal überhaupt in einem gedrucktem Werk erscheint ${ }^{8}$ :

Item ein Sack pfeffer wigt 2 ct $1 / 2$ - $9 \mathrm{lb}$ vnd ist $1 \mathrm{lb}$ kaufft worden pro $8 ß-3$ hel vnd sol fur den sack abschlahen $3 \mathrm{lb}+3 / 4$ [...] (ebd.: fol. $\left.88^{\mathrm{v}}\right)$.

Den Ansatz des Dreisatzes formuliert Widmann im Gegensatz zu Ries durchgehend umgangssprachlich. Für die Grundrechenarten, die Mischrechnung, 
den Kettensatz, die Gesellschaftsrechnung und die regula falsi gibt er in den Aufgaben dagegen Notationsschemata an. Diese weichen allerdings zum Teil voneinander $\mathrm{ab}$, insbesondere bei der Mischrechnung, wo Widmann mehrere horizontale und vertikale Schemata anführt (Widmann 1489: fol. 102 ${ }^{\mathrm{v}}, 103^{\mathrm{r}}$, $\left.110^{\mathrm{r}}, 160^{\mathrm{r}}, 161^{\mathrm{r}}\right)$.

Wie Ries verwendet auch Widmann gleichberechtigt deutsche und lateinische Fachbegriffe:

[...] so subtrahir 3 von 10 pleyben 6 das diuidir mit 1 facit 7 apfel pro $1 \mathrm{Nu}$ des gleichen nym 2 von 30 vnnd diuidir mit 4 facit 7 Auch nym 1 vonn 50 pleibt 49 das teyl in 7 facit 7 apfel Und ist gemacht. (ebd.: fol. 134 ${ }^{\mathrm{r}}$ )

Anders als Ries führt Widmann jedoch häufig für nur umständlich zu umschreibende Begriffe - wie zum Beispiel für Differenz, Produkt und Quotient - die lateinischen Fachausdrücke ein (Gärtner 2000: 536-568).

Keines der Sprachmittel und keines der Notationsschemata in Ries' Rechenbuch war für sich genommen neu. Diese folgten dem Stil der libri d'abbaco und der frühen deutschen Rechenbücher. Die besondere didaktische Qualität der Sprache in der Rechenung auf der linihen vnd federn lag vielmehr in der umfassenden und offensichtlich bewussten Reduktion von Sprachstil, Signalwörtern und Fachvokabular sowie in der Beschränkung auf einige wenige, aber einheitlich ausgeführte Notationsformen. Widmanns Buch zeigte hingegen deutlich mehr Vielfalt bei Satzbau, Wortschatz und Notationsschemata. Das lässt sich auch in vielen anderen Rechenbüchern, etwa im neu geordnet Rechenbuchlein von Johann Böschenstain (1472-1540) und im Rechenbuch Auff Linien und Ziffern von Jacob Köbel (um 1462-1533) deutlich feststellen (Böschenstain [1518] 1983, Köbel 1549). Der Leser konnte sich bei Ries schneller innerhalb der Lehrtexte orientieren und stand einer deutlich kleineren Menge an zu lernenden Fachwörtern und Notationsschemata gegenüber. Mit seiner reduzierten Wortwahl und Syntax bei Definitionen und Rechenaufgaben löste sich Ries deutlich mehr als andere Autoren von Rechenbüchern von der humanistisch geprägten Vorstellung des einfachen, aber eleganten genus subtile der Fachprosa zu Gunsten eines einfacheren Zugangs zum Lehrstoff.

\section{Ries' Didaktik: Grundlage seiner Popularität}

Was hob die Didaktik in Ries' zweitem Rechenbuch von den Werken zeitgenössischer Rechenmeister und Mathematiker so ab, dass es sie in der Verbreitung weit hinter sich ließ? Im Vergleich mit Johannes Widmann Behende vnd hubsche Rechenung lässt sich die Antwort darauf so zusammenfassen: 
Bei der fachlichen Auswahl des Lehrstoffs entschied sich Ries für einen Kompromiss zwischen der Aufnahme tradierter Elemente der Rechenkunst und dem tatsächlichen Bedarf der Kaufleute und Handwerker. Die von seinem Publikum noch oft verwendete Linienrechnung, eigentlich überflüssige, aber einfach zu verstehende Rechenarten und die leicht abgrenzbare Unterhaltungsmathematik nahm er daher in sein Buch auf. Schwierige und in der Praxis nicht notwendige Rechenarten und Regeln fielen dagegen weg. Die Rechenregeln und -beispiele der ,Practica' ordnete Ries nicht nach einer abstrakten Systematik, sondern nach den praktischen Anwendungsgebieten seines Publikums. Für eine angemessene Erklärung der besonderen didaktischen Qualität der Rechenung auf der linihen vnd federn reicht diese schon von Unger (1888: 51), Cantor (1900: 421-422), Deschauer (1992: 9-10) und Wußing (2009: 61) angesprochene besondere Auswahl und Anordnung des Lehrstoffs alleine jedoch nicht aus. Das durchaus populäre Rechenbuch von Jacob Köbel Rechenbuch Auff Linien und Ziffern (1549) bot etwa ähnliche Inhalte und eine ebenfalls anwendungsorientierte Präsentation, erreichte aber trotzdem nie den Verbreitungsgrad von Ries' zweitem Rechenbuch.

Die zweite Besonderheit von Ries' Rechenbuch, die es etwa von Köbels Werk abhob, lag im flexiblen Umgang mit dem etablierten Aufbau der Lehrtexte in den damaligen Rechenbüchern. Die Erläuterungen der Rechenarten und -regeln folgten zwar dem gängigen Schema der damaligen Rechenbücher. Anders als bei Widmann und den meisten anderen Rechenbuchautoren waren die Bestandteile seiner Erläuterungen - Definition, Erklärung der Rechenschritte und Kurzbeispiel - jedoch so kompakt gehalten, dass sie als Einheit gelesen wurden und damit eine hohe Verständlichkeit des Textes bewirkten. Auch bei der Gliederung der Übungsaufgaben in der ,Practica' folgte Ries zwar dem gängigen Muster anderer Rechenbücher. Im Gegensatz zu Widmann und den meisten anderen Autoren stellte er die Lösung jedoch - gut auffindbar gleich hinter die Aufgabe und passte überdies den Detaillierungsgrad der Beschreibungen dem Kontext des Rechenbuchs an: Bekanntes wurde verkürzt erläutert, Neues dagegen ausführlich und mit Rechenvorteilen beschrieben (Deschauer 1992).

Als dritter und ebenso wichtiger Punkt ist der schon von Gärtner (2000: 213-217) angesprochene besondere Sprachstil von Ries zu nennen, mit dem er die humanistische Vorstellung einer einfachen, aber stilvollen Fachprosa zumindest teilweise aufgab. Im Grundsatz ging Ries in der Auswahl seiner Stilmittel nicht über Widmann und andere Autoren hinaus, etwa bei der imperativen Ansprache des Lesers und der Verwendung lateinisch-deutschen Fachvokabulars. Er reduzierte jedoch im Vergleich zu Widmann den ohnehin beschränkten Sprachstil der Rechenbücher noch weiter. In wiederkehrenden Textarten wie Definitionen und Rechenbeispielen wurden Syntax und Vokabular weitgehend $\mathrm{zu}$ Stereotypen und boten so dem Leser eine leichtere Orientierung im Text. 
Zweifelsohne war Ries' Rechenung auf der linihen vnd federn im Vergleich zu Widmanns Behende vnd hubsche Rechenung auff allen kauffmanschafft das bessere Lehrbuch für Kaufleute und Handwerker der frühen Neuzeit. Auch gegenüber anderen Werken haben die gemachten Beobachtungen zur didaktischen Qualität von Ries' zweitem Rechenbuch Bestand. Insbesondere Ries flexibler Umgang mit der Struktur der Lehrtexte und sein reduzierter Sprachstil hoben ihn auch von den anderen populären Autoren wie etwa Böschenstain und Köbel ab. Johann Albert (1488-1558), um ein anderes Beispiel zu nennen, folgte in seinem New Rechenbüchlein auff der Federn (Albert 1544) zwar dem reduzierten Stil von Adam Ries und dessen flexiblen Umgang mit den einzelnen Teiltexten. Seine Einführung in die Linienrechnung geriet ihm aber so lang und vollständig, dass er sein Rechenbuch in zwei getrennte Bücher gliederte, eines zur Linien- und eines zur schriftlichen Rechnung.

Dennoch bleibt die Frage offen: War es nur die didaktische Güte, die Ries' zweites Rechenbuch so vor allen anderen hervorhob? Hier ist mit Pierre Jeannin (2001) vor allem nach der Rolle seiner Drucker und Verleger zu fragen, die generell eine aktive Rolle bei der Gestaltung und dem Vertrieb der Rechenbücher übernahmen. Ob Ries' Verleger aber hauptsächlich für dessen Erfolg als Rechenbuchautor verantwortlich sind, erscheint fraglich. Seine beiden ersten Drucker, Matthes Maler und Melchior Sachse d. Ä, waren zwar bedeutende Drucker in der Universitätsstadt Erfurt, ohne aber auf Fachprosa spezialisiert zu sein (Benzing 1982: 109-110). Erst mit dem Wechsel zu Christian Egenolff in Frankfurt am Main 1533 geriet Ries an einen Drucker, der sich auf Fachprosa, darunter auch Rechenbücher, spezialisiert hatte (Jeannin 2001: 71-72, Benzing 1982: 120-121). Zu diesem Zeitpunkt hatte Ries' zweites Rechenbuch aber schon beachtliche sieben Auflagen hinter sich (Hoock 1991: 193-207). Offensichtlich fanden hier ein bereits erfolgreicher Fachautor und ein ebenso erfolgreicher Drucker mit einer passenden fachlichen Ausrichtung zusammen. Zudem druckte Egenolff nicht nur Ries' Rechenbuch, sondern auch die von zahlreichen anderen Autoren, darunter Köbel und Albert (Jeannin 2001: 71-72). Letztendlich muss es doch vor allem das besondere didaktische Geschick des Adam Ries gewesen sein, die sein Rechenung auf der linihen vnd federn zum populärsten deutschen Rechenbuch der frühen Neuzeit machte.

\section{Anmerkungen}

1 Auf dem Abakus-ähnlichen Rechentisch bzw. Rechentuch befinden sich Linien für die aufsteigenden Zehner-Potenzen: Eins, Zehn, Hundert, usw. Die Zwischenräume zwischen den Linien stehen für die Fünferbündel: Fünf, Fünfzig, Fünfhundert, usw. Die Zahlen werden mit ,Rechenpfennigen' oder ,Rechensteinen' auf oder zwischen den Linien abgelegt. Zwei Rechensteine auf der Zehnerlinie bedeuten zum Beispiel 20. Addieren und 
Subtrahieren lassen sich dann auf das Abzählen der Rechensteine und das Bündeln bzw. Entbündeln zurückführen: Zehn Steine auf einer Linie werden zu einem Stein auf der nächsthöheren ,gebündelt' und umgekehrt (Unger 1888: 66-70).

2 Bei der regula falsi werden jeweils zwei beliebige Werte für die gesuchten Variablen in die Gleichungen eingesetzt. Aus den Differenzen der so gewonnenen Ergebnisse ('Lügen') mit dem richtigen Ergebnis lässt sich dann die tatsächliche Lösung berechnen (Tropfke 1980: 180-185).

3 In allen Zitaten sind die im Frühneuhochdeutschen üblichen Abkürzungen und Grapheme aufgelöst: Ein überstrichenes ,m' wird $\mathrm{zu}, \mathrm{em}$ ' oder ,mm', ein ,e über dem ,a' wird zu ,ae‘, usw. (Reichmann/Wegera 1993: \$L5, 12, 15, 16).

4 Die Zechrechnung behandelt die Lösung unbestimmter und ganzzahliger linearer Gleichungssysteme, für die Ries den Gauß'schen Algorithmus angibt (Deschauer 1992: 184-188). Eine typische Aufgabe lautet: „21 Personen - Männer und Frauen - haben 81 Pfennig vertrunken. Jeder Mann soll 5 Pfennig und jede Frau 3 Pfennig geben. Nun frage ich, wie viele Personen beiderlei Geschlechts es im einzelnen gewesen sind?" (Übertragung ins Hochdeutsche: ebd.: 104).

5 Die Aufgabe lautet: „Eine Schnecke befindet sich 32 Ellen tief in einem Brunnen. Jeden Tag kriecht sie $4 \frac{2}{3}$ Ellen herauf und fällt jede Nacht 33/4 Ellen zurück. In wie vielen Tagen kommt sie heraus?" (Übertragung ins Hochdeutsche: Deschauer 1992: 107).

6 Anders als heute multiplizieren Ries und die anderen Rechenmeister beim Dreisatz zunächst und dividieren anschließend. Dieses Verfahren erschwert zwar das Verständnis des Rechenwegs, vermeidet aber, soweit möglich, das Rechnen mit Brüchen.

7 Widmann und Ries notieren bei Brüchen Zähler und Nenner übereinander, während sie hier für einen besser lesbaren Satz nebeneinander angeordnet sind.

8 Kaunzner (1968: 14-15) führt Widmanns Schreibweisen für Addition und Multiplikation darauf zurück, dass das Plus- und Minuszeichen den Kaufleuten schon als Kennzeichnungen von Über- und Untergewicht einer Ware bekannt waren. Cantor (1900: 230-231) lehnt diese schon früh geäußerte These zu Herkunft von Plus- und Minuszeichen ab und verweist auf eine spätere Aufgabenstellung bei Widmann („Eyner hat kaufft 6 Eyer - $2 \mathrm{~d}$ [Pfennig] pro $\left.4 \mathrm{~d}+1 \mathrm{ey}^{\prime \prime}\right)$, die dem Schema des Über- und Untergewichts einer Ware offensichtlich nicht genügt. Wahrscheinlich hat Widmann jedoch tatsächlich die kaufmännische Tradition des Plus- und Minuszeichen genutzt und als Mathematiker dann umgehend von der Herleitung abstrahiert.

\section{Literatur}

Albert, Johann, 1544. New Rechenbüchlein auff der Federn gantz leicht, aus rechtem grund, jnn Gantzen vnd Gebrochen, Neben angehefftem, vnlangst, ausgelassnem Büchlein auff den Linien, dem einfeltigen gemeinen Man, vnd anhebenden der Arithmetica Liebhabern zu gut. Wittenberg: Georg Rhau [Digitalisat der Sächsische Landesbibliothek - Staats- und Universitätsbibliothek Dresden, http://digital.slub-dresden.de/ppn276661370, 9.9.2010].

Alten, Heinz-Wilhelm u. a., 2003. 4000 Jahre Algebra. Geschichte, Kulturen, Menschen. Berlin u. a.: Springer.

Benzing, Josef, 1982. Die Buchdrucker des 16. und 17. Jahrhunderts im deutschen Sprachgebiet. 2. verbesserte und ergänzte Auflage.Wiesbaden: Harrasowitz. [= Beiträge zum Buch- und Bibliothekswesen, 12].

Böschenstain, Johannes, [1518] 1983. Ain neu geordnet Rechenbüchlein mitt den zyffern. den angenden Schülern zu nutz. Nachdruck der 3. Aufl., Augsburg 1518. Bearbeitet von Wolfgang Meretz. Berlin: BGJ Drucktechnik.

Busard, Hubert L.L., 1997. Über die Entwicklung der Mathematik in Westeuropa zwischen 1100 und 1500. NTM. Internationale Zeitschrift für Geschichte und Ethik der Naturwissenschaften und der Medizin, 5, 211-235.

Cantor, Moritz, 1900. Vorlesungen über Geschichte der Mathematik. Bd. 2. Von 1200 bis 1668. 2. Aufl.. Leipzig: Teubner. 
Deubner, Fritz, 1992. ... nach Adam Ries. Leben und Wirken des großen Rechenmeisters. Überarbeitet und ergänzt von Wolfgang Riemer. Taucha: Tauchaer Verlag.

Deschauer, Stefan, Hg., 1991 [1522]. Das 2. Rechenbuch von Adam Ries. Nachdruck der Erstausgabe Erfurt 1522 mit einer Kurzbiographie, bibliographischen Angaben und einer Übersicht über die Fachsprache. München: Institut für Geschichte der Naturwissenschaften. [= Algorismus, 5].

Deschauer, Stefan, 1992. Das zweite Rechenbuch von Adam Ries. Eine moderne Textfassung mit Kommentar und metrologischem Anhang und einer Einführung in Leben und Werk des Rechenmeisters. Braunschweig: Vieweg.

Deschauer, Stefan, 2003. Das 2. Rechenbuch von Adam Ries. Ein Bestseller für das Wirtschaftsleben des 16. Jahrhunderts. In: Jürgen Kiefer und Karin Reich, Hg., Gemeinnützige Mathematik: Adam Ries und seine Folgen, Erfurt: Akademie gemeinnütziger Wissenschaften zu Erfurt, 9-31. [=Acta Academiae Scientiarum, 8].

van Egmond, Warren, 1980. Practical Mathematics in the Italian Renaissance: A Catalog of Italian Abbacus Manuscripts and Printed Books to 1600. Firenze: Instituto e Museo Di Storia Della Scienza.

Eichler, Birgit, 1992. Wissenstransfer und Sprachgebrauch in Adam Rieses Schriften. In: Rainer Gebhardt, Hg., Adam Ries - Humanist, Rechenmeister, Bergbeamter. Beiträge zum wissenschaftlichen Kolloquium am 18. Juli 1992 in Annaberg-Buchholz. Annaberg-Buchholz: Adam-Ries-Bund, 93-100. [= Schriften des Adam-Ries-Bundes, 1].

Eis, Gerhard, 1967. Mittelalterliche Fachliteratur, 2. Aufl., Stuttgart: Metzler.

Endres, Rudolf, 1982. Sozial- und Bildungsstrukturen fränkischer Reichsstädte im Spätmittelalter und in der frühen Neuzeit. In: Horst Brunner, Hg., Literatur in der Stadt. Bedingungen und Beispiele städtischer Literatur des 15. bis 17. Jahrhunderts, Göppingen: Kümmerle, 37-72. [= Göppinger Arbeiten zur Germanistik, 343].

Endres, Rudolf, 1996. Handwerk - Berufsbildung. In: Christa Berg u. a., Hg., Handbuch der deutschen Bildungsgeschichte. Bd. I. 15. bis 17. Jahrhundert. Von der Renaissance und der Reformation bis zum Ende der Glaubenskämpfe. München: Beck, 375-424.

Folkerts, Menso, 2003. Die Ausbildung von Rechenmeistern: Dargestellt an ausgewählten Beispielen. In: Jürgen Kiefer und Karin Reich, Hg., Gemeinnützige Mathematik. Adam Ries und seine Folgen. Erfurt: Akademie gemeinnütziger Wissenschaften zu Erfurt, 89-129. [=Acta Academiae Scientiarum, 8].

Folkerts, Menso, 2008. Die Faßmessung (Visierkunst) im späten Mittelalter und in der frühen Neuzeit. In. Rainer Gebhardt, Hg., Visier- und Rechenbücher der frühen Neuzeit. Tagungsband zum Wissenschaftlichen Kolloquium "Visier- und Rechenbücher der Frühen Neuzeit" vom 18.-20. April 2008 in der Berg- und Adam-Ries-Stadt Annaberg-Buchholz. AnnabergBuchholz: Adam-Ries-Bund, 1-36. [= Schriften des Adam-Ries-Bundes, 19].

Folkerts, Menso/Reich, Karin, 2001. Rechenmeister. In: Menso Folkerts u. a., Hg., Mass, Zahl und Gewicht. Mathematik als Schlüssel zu Weltverständnis und Weltbeherrschung. 2. Aufl., Wiesbaden: Harrassowitz, 189-216. [= Ausstellungskataloge der Herzog-August-Bibliothek, 60].

Gärtner, Barbara, 2000. Johannes Widmanns „Behende vnd hubsche Rechenung“. Die Textsorte "Rechenbuch" in der Frühen Neuzeit. Tübingen: Niemeyer. [= Reihe Germanistische Linguistik, 222].

Gebhardt, Rainer, Hg., 1994. Einblicke in die Coß von Adam Ries. Eine Auswahl aus dem Original mit aktuellen Anmerkungen und Kommentaren. Stuttgart, Leipzig: Teubner und Zürich: Verlag der Fachvereine.

Gebhardt, Rainer, 2003. Die gedruckten Rechenbücher des Adam Ries. Übersicht und neue Funde. In: Jürgen Kiefer und Karin Reich, Hg., Gemeinnützige Mathematik. Adam Ries und seine Folgen. Erfurt: Akademie gemeinnütziger Wissenschaften zu Erfurt, 33-53. [=Acta Academiae Scientiarum, 8].

Gericke, Helmuth, 1980. Mathematik im Abendland. Von den römischen Feldmessern bis zu Descartes. Berlin u. a.: Springer.

Giesecke, Michael, 2006. Der Buchdruck in der frühen Neuzeit. Eine historische Fallstudie über die Durchsetzung neuer Informations- und Kommunikationstechnologien. 4. Aufl., Frankfurt a. M.: Suhrkamp.

Grosse, Hugo, [1901] 1965. Historische Rechenbücher des 16. und 17. Jahrhunderts und die Entwicklung ihrer Grundgedanken bis zur Neuzeit. Ein Beitrag zur Geschichte der Methodik des Rechenunterrichts. Neudr. d. Ausg. Leipzig 1901. Wiesbaden: Sändig. 
Günther, Siegmund, 1969 [1887]. Geschichte des mathematischen Unterrichts im deutschen Mittelalter bis zum Jahre 1525. Unveränderter Neudruck der Ausgabe von 1887. Wiesbaden: Sändig. [= Monumenta Germaniae Paedagogica, 3].

Haage, Bernard Dietrich/Wegner, Wolfgang, 2007. Deutsche Fachliteratur der Artes in Mittelalter und Früher Neuzeit. Berlin: Schmidt. [= Grundlagen der Germanistik, 43].

Habermann, Mechthild, 2001. Deutsche Fachtexte der frühen Neuzeit. Naturkundlich-medizinische Wissensvermittlung im Spannungsfeld von Latein und Volkssprache. Berlin/New York: de Gruyter. [= Studia linguistica Germanica, 61].

Hanschmidt, Alwin, 2005. Elementarbildung und Berufsausbildung 1450 bis 1750. Inhalte und Institutionen. In: Alwin Hanschmidt und Hans-Ulrich Musolff, Hg., Elementarbildung und Berufsausbildung, 1450-1750, Köln u. a.: Böhlau, 19-46.

Hoock, Jochen, Hg, 1991. Ars Mercatoria. Handbücher und Traktate für den Gebrauch des Kaufmanns 1470-1820. Eine analytische Bibliographie. Band 1. 1470-1600. Paderborn: Schöningh.

Høyrup, Jens, 2007. Jacopo da Firenze's Tractatus algorismi and early Italian abbacus culture. Basel: Birkenhäuser. [=Science Networks Historical Studies, 34].

Isenmann, Eberhard, 1988. Die deutsche Stadt im Spätmittelalter 1250-1500. Stadtgestalt, Recht, Stadtregiment, Kirche, Gesellschaft, Wirtschaft. Stuttgart: Ulmer.

Isenmann, Eberhard, 2004. Die Bedeutung der Sozial- und Wirtschaftsgeschichte fur die Allgemeine Geschichte des Mittelalters. In: Günther Schulz u. a., Hg., Sozial- und Wirtschaftsgeschichte. Arbeitsgebiete - Probleme - Perspektiven. 100 Jahre Vierteljahrschrift für Sozial-und Wirtschaftsgeschichte). Wiesbaden: Steiner, 469-524. [= Vierteljahrschrift für Sozial- und Wirtschaftsgeschichte, Beihefte, 169].

Jeannin, Pierre, 2001. Vertrieb und Verbreitung der Handbücher. Funktionen und Strategien des Verlagssektors. In: Jochen Hoock und Pierre Jeannin, Hg., Ars Mercatoria. Handbücher und Traktate für den Gebrauch des Kaufmanns, 1470-1820. Eine analytische Bibliographie in 6 Bänden. Bd. 3: Analysen (1470-1700). Paderborn: Schöningh, 37-89.

Kaunzner, Wolfgang, 1968. Über Johannes Widmann von Eger. Ein Beitrag zur Geschichte der Rechenkunst im ausgehenden Mittelalter, o. O. [München]. [= Veröffentlichungen des Forschungsinstituts des Deutschen Museums für die Geschichte der Naturwissenschaft und der Technik, Reihe C, Quellentexte und Übersetzungen, 7].

Kaunzner, Wolfgang, 1996. Johannes Widman, Cossist und Verfasser des ersten großen deutschen Rechenbuchs. In: Rainer Gebhardt, Hg., Rechenmeister und Cossisten der frühen Neuzeit. Beiträge zum wissenschaftlichen Kolloquium am 21. September 1996 in Annaberg-Buchholz. Annaberg-Buchholz: Adam-Ries-Bund, 37-51.

Köbel, Jacob,1549. Rechenbuch Auff Linien und Ziffern Mit einem Visir Büchlin Klar unnd verstendtlich fürgeben. Frankfurt: Christian Egenolff [Bayerische Staatsbibliothek, Res/Math.p. 744 r, Digitalisat der BSB: http://daten.digitale-sammlungen.de/bsb00028755/image_1, 9.9.2010].

Neddermeyer, Uwe, 1998. Von der Handschrift zum gedruckten Buch. Schriftlichkeit und Leseinteresse im Mittelalter und in der frühen Neuzeit. Quantitative und qualitative Aspekte. Bd. 1 Text. Wiesbaden: Harrasowitz. [= Buchwissenschaftliche Beiträge aus dem Deutschen Bucharchiv München, 61].

Osterwalder, Fritz, 2004. Methode. In: Dietrich Benner und Jürgen Oelkers, Hg., Historisches Wörterbuch der Pädagogik. Weinheim, Basel: Beltz, 638-659.

Reichmann, Oskar/Wegera, Klaus-Peter, Hg., 1993. Frühneuhochdeutsche Grammatik. Tübingen: Niemeyer. [Sammlung kurzer Grammatiken germanischer Dialekte: A, Hauptreihe, 12].

Ries, Adam, 1522. Rechenung auf der linihen vnd federn. Erfurt: Matthes Maler [Faksimile in: Deschauer, Stefan, Hg., 1991. Das 2. Rechenbuch von Adam Ries. Nachdruck der Erstausgabe Erfurt 1522 mit einer Kurzbiographie, bibliographischen Angaben und einer Übersicht über die Fachsprache. München: Institut für Geschichte der Naturwissenschaften. [= Algorismus, 5].

Ries, Adam, 1548. Rechnung auff der Linien und Federn. Leipzig: Michel Blum. [Sächsische Landesbibliothek - Staats- und Universitätsbibliothek Dresden, Math.1015, misc.2, Digitalisat der SLUB: http://digital.slub-dresden.de/ppn273513583, 9.9.2010].

Schneider, Ute, 2005. Das Buch als Wissensvermittler in der Frühen Neuzeit. In: Johannes Burkhardt und Christine Werkstetter, Hg., Kommunikation und Medien in der Frühen Neuzeit. München: Oldenbourg, 63-78. [= Historische Zeitschrift, 41].

Tropfke, Johannes, 1980, Geschichte der Elementarmathematik. Bd. 1, Arithmetik und Algebra. 4. Aufl., vollständig neu bearbeitet von Kurt Vogel, Karin Reich und Helmuth Gericke. Berlin/ New York: de Gruyter. 
Unger, Friedrich, 1888. Die Methodik der praktischen Arithmetik in historischer Entwickelung vom Ausgange des Mittelalters bis auf die Gegenwart. Nach den Originalquellen bearbeitet. Leipzig: Teubner.

Vogel, Kurt, Hg, 1954. Die Practica des Algorismus Ratisbonensis. Ein Rechenbuch des Benediktinerklosters St. Emmeram aus der Mitte des 15. Jahrhunderts nach den Handschriften der Münchner Staatsbibliothek und der Stiftsbibliothek St. Florian. München: Beck.

Vogel, Kurt, Hg, 1980. Das Bamberger Blockbuch. Inc. typ. Ic. I 44 der Staatsbibliothek Bamberg. Ein xylographisches Rechenbuch aus dem 15. Jahrhundert. Hg. und erläutert von Kurt Vogel. Mit einer buchkundlichen Beschreibung von Bernhard Schemmel. München u. a.: Saur.

Wappler, Emil, 1900. Zur Geschichte der Mathematik im 15. Jahrhundert. Zeitschrift für Mathematik und Physik, Historisch-litterarische Abteilung, 45, 47-51.

Widmann, Johannes, 1489. Behende vnd hubsche Rechenung auff allen kauffmanschafft. Leipzig: Konrad Kachelofen [Bayerische Staatsbibliothek, Inc.c.a. 82. Zit. nach: Digitalisat der BSB: http://daten.digitale-sammlungen.de/bsb00028755/image_1, 9.9.2010].

Wittmann, Reinhard, 1999. Geschichte des deutschen Buchhandels. 2. Aufl., München: Beck.

Wolff-Thomsen, Ulrike, 2001. Titelgrafiken in deutschen und niederländischen Kaufmannsbüchern. In: Jochen Hoock und Pierre Jeannin, Hg., Ars Mercatoria. Handbücher und Traktate für den Gebrauch des Kaufmanns, 1470-1820. Eine analytische Bibliographie in 6 Bänden. Bd. 3: Analysen (1470-1700). Paderborn: Schöningh, 173-192.

Wußing Hans, Hg. 2008. 6000 Jahre Mathematik. Eine kulturgeschichtliche Zeitreise Bd. 1. Von den Anfängen bis Leibniz und Newton. Berlin/Heidelberg: Springer.

Wußing, Hans, 2009. Adam Ries. 3. Aufl., Leipzig: Edition am Gutenbergplatz.

Peter Gabriel

Schauflerpfad 25

13503 Berlin

Deutschland

E-Mail: peter.gabriel@berlin.de 